\title{
Subatomic Physics Education and Outreach in Newfoundland
}

\author{
Svetlana Barkanova* \\ Memorial University of Newfoundland, Grenfell Campus, Corner Brook, Canada \\ E-mail: sbarkanova@grenfell.mun.ca
}

\begin{abstract}
A physics education provides students with a valuable and flexible skill set that opens doors to a wide variety of career paths, including industry, education and finance. However, Physics is almost universally unpopular in our local schools, especially among girls. This paper describes a long-term scientific and cultural outreach program we developed for youth in Newfoundland, Canada, especially rural youth, girls, and Indigenous students. We feature female and Indigenous role models, engage Indigenous storytelling, discuss a wide range of career opportunities, and emphasize a diverse set of skills required in modern science, such as cooperation and communication. As these students enter our post-secondary physics program, which is specifically developed to focus on subatomic physics and astrophysics, we engage them in research as early as their second year, making sure they are well prepared to continue at graduate schools in both theory and experiment.
\end{abstract}

XXIX International Symposium on Lepton Photon Interactions at High Energies - LeptonPhoton2019 August 5-10, 2019

Toronto, Canada

${ }^{*}$ Speaker. 


\section{Motivation}

Currently, in Canada, only about $20 \%$ of bachelor's degrees in physics are held by women [1]. The large variations in the percentage of female physicists in different countries suggest that culture has a large influence on the participation of women in physics, with Canada along with the US and Japan ranking near the bottom [2]. The situation is similar with Indigenous students, with a very small fraction choosing physics-related careers. With most of the rural youth in our region never even meeting a physicist, it is no wonder most of them would not consider physics as a viable profession. Yet, a physics background is required in many science-related careers, so our primary focus is science outreach in physics, especially for girls, rural youth, and Indigenous students. In economies that are based on innovation and new technology, recruitment and retention of talent in the sciences and engineering are crucial. Women, rural youth, and Indigenous students constitute an enormous potential resource for both industry and academia, so increasing the participation of these under-represented groups in science and engineering will tap more fully the potential of the talents of our population. In general, there is lower than average interest in university education in our region, and only $16 \%$ of Corner Brook population has university certificate, diploma or degree at bachelor level or above, in comparison, for example, to $29 \%$ in St. John's [3]. One of the reasons may be that most of the local high school students are reluctant to leave the island or move to the main campus of Memorial University of Newfoundland (MUN) in St. John's (population 109,000 [3], especially students from smaller communities. Now, they do not have to: the second largest MUN campus, Grenfell Campus in Corner Brook (population 19,800 [3]), has expanded many of its programs, in particular opening a BSc program in Physics, with a focus specifically on subatomic physics and astrophysics. The program was an immediate success, probably due to its novelty, but our next challenge is making sure that students stay, and we do this by creating engaging, inclusive, and comfortable environment. We are also now offering $\mathrm{PhD}$ and MSc programs in theoretical particle physics via St. John's campus, the first graduate program in subatomic physics in Newfoundland.

\section{Methodology: Outreach}

There are three main objectives in our science outreach program. First, given that our target demographic resides in a formerly resource-based economy, we aim to support future innovation and economic diversification by attracting local youth to science-related careers through the promotion of natural sciences in Western Newfoundland, particularly to youth in rural areas, females, and Indigenous students. Second, we build on the complementary knowledge, expertise, and resources at MUN's Grenfell Campus, the Qalipu First Nation, and Parks Canada to develop a long-term, sustainable scientific and cultural outreach program on campus, in schools, and in national and provincial parks. And, third, we coordinate our science outreach content with the existing provincial school curriculum, develop resources for teachers, and participate in professional teacher development, especially in the areas related to quantum physics, subatomic physics, astrophysics and cosmology. Since there is a significant body of research on factors affecting young women's career choices, but much less data on Indigenous and rural youth, we build on the common assumption that "what works for women works for everyone", for now, while we try to 
identify local factors influencing the participation rates of underrepresented groups in science. Research shows $[4,5,6]$ that the future ability to be able to help society is one of the deciding factors affecting young women's career choices. Another deciding factor affecting young women's career choices is having a job where they can work with people, rather than things, and express themselves creatively. Thus, we introduce science through a creative and historical lens, feature female physicists, include context-relevant examples of females and minorities as successful scientists, engage Indigenous story-telling, emphasize the beauty of our universe, showcase the creativity involved in doing physics, discuss career opportunities, and emphasize a diverse set of skills required in modern science such as cooperation, communication and emotional intelligence. For the general public events, we can choose any content and often focus on many fascinating topics of subatomic physics; however, schools that bring their students on campus, or invite our team to visit their classes, require us to cover their curriculum, and there is no subatomic physics in the provincial school program. We address this gap in two ways: first, the presenter, who is either a faculty member or a physics major with research experience in subatomic physics, briefly outlines her own experiences and career choices during the introductions; and, second, we build on the curriculum-related material to be presented, going from astronomy to Dark Matter to Physics Beyond Standard Model, for example. Our action plan is basically three-pronged, focusing on activities centered around youth programs on campus, in schools, and public events on campus and in Gros Morne National Park. On campus, the youth groups (mostly Grade 6 and older) are split into smaller sub-groups, up to 15 students in each, and rotated through various age-appropriate activities, such as the observatory tours, short lectures, and hands-on exercises or experiments. The Grenfell Observatory, which houses several telescopes including Ritchey-Chretien Cassegrain reflector with the $60 \mathrm{~cm}$ main mirror under its 6-metre dome, is a major attraction in the region, and many local schools bring their student to campus on a regular basis. Our team's visits to schools are harder to organize, but we strive to visit about ten Western Newfoundland schools, mostly rural, per year, with curriculum-relevant lectures and demos supplemented by the cultural content where appropriate; we also develop and provide resources for teachers. And, finally, several times per year, we have very popular (fully booked with prior registration on Eventbrite) public events on campus and community events related to science and dark sky at Gros Morne National Park organized jointly with Parks Canada. A high percentage of MUN students are Indigenous, especially in Corner Brook which has 24\% Indigenous population [3], and many of them have been helping with our science outreach activities. The Qalipu First Nation, our community partners on this science-outreach project, collect relevant Indigenous knowledge such as stories related to the sky, incorporate them into some of our programs, and collaborate on school visits and public events. The "Muin and the Seven Bird Hunters" (MuinaqqL'uiknekTe'sijikNtuksuinu'k) story [7], for example, can be read by one of our community partners or Grenfell student volunteers while kids are following the motion of Muin, a bear chased by birds (Ursa Major) around Tatapn (Polaris) with Star Wheels. Qalipu is a Mi'kmaq First Nation established in 2011 as an Indigenous Band under the Indian Act, and, with some 24,000 members spread across many communities, Qalipu is one of the largest First Nation groups in Canada. Qalipu has no reserve land and no separate school system; it is made up of 66 traditional Mi'kmaq communities located in western and central Newfoundland [8]. The staff of the Qalipu First Nation head office in Corner Brook are especially instrumental in integrating the Indigenous knowledge in our science curriculum and establishing contacts with 
schools. The impact of our programs is evaluated by multiple-choice question polls which are scanned and processed statistically, showing significant increase in interest in science-related careers as a result of participating in our programs. We have also learned that incorporating Mi'kmaq culture attracts a significant number of Indigenous participants to our public events, and that most of our participants are strongly in favor of more science-related events in the future. Outreach is not the same as recruitment, of course, and recruitment is not one of the goals of our program. We did observe a strong increase in student numbers in our physics courses, though, which is probably a combination of physics promotion, introduction of a wider variety of second-year courses, and increased popularity of a new BSc program in Physics which is focused specifically on subatomic physics and astrophysics.

\section{Methodology: Retention}

With the graduation rates at Grenfell Campus of MUN quite below the national average, we have to plan especially carefully to make sure our students stay. We approach this in two ways: by introducing an engaging set of physics courses at the second-year level and creating inclusive and engaging environment for our students by providing research opportunities and encouraging networking and collaborative projects. Ideally, we would like to introduce subatomic physics and astrophysics content as soon as possible, but we do not have the freedom to alter the first-year curriculum due to the requirements of other programs such as engineering. Thus, along with the standard set of second-year undergraduate courses such as Modern Physics and Computational Mechanics, we offer one course in Subatomic Physics (introductory nuclear and particle physics with applications such as energy and medicine; followed by Particle Physics course at the third-year level) and two courses in Astronomy and Astrophysics. After the second year, students are offered various research opportunities, mostly in particle physics, provided with funding by MUN, the undergraduate student research awards by the Natural Sciences and Engineering Research Council of Canada (NSERC USRA), summer undergraduate research experience funding by CERN and Canada's Institute of Particle Physics (IPP), undergraduate research scholarships by the Canadian Institute of Nuclear Physics (CINP), and NSERC grants of faculty supervisors. For example, in 2014, Jessica Strickland, became the first MUN student to win the IPP/CERN research fellowship to spend a summer working at CERN [9, 10]; in 2018, Mallory Snow was the first to be supported by CINP [11]. The accomplishments of both women were widely publicized in the local press. "“'When I first came to Grenfell I really wanted to go into education..." said Strickland. ... "Then I realized that I really loved research."' [10]. She is currently a $\mathrm{PhD}$ student at the University of Twente, the Netherlands, and gives seminars to our undergraduate students when she visits. The undergraduate students are encouraged to collaborate where appropriate, help with the science outreach $[12,13]$, present student seminars on topics relevant to their research field in both theory and experiment, network [14], and investigate future study and career opportunities in subatomic physics. So far, we had a $100 \%$ graduation rate in four years, but it is too early to say if this trend will continue. Most of our graduates are currently continuing in $\mathrm{PhD}$ and MSc programs in Canada and abroad; one chose to continue to study theoretical particle physics at our St. John's MUN campus. Our other graduate students, mostly international, who take their courses at St. John's campus for a year and then come to Corner Brook to do research, also act as part of our support 
system for the undergraduate program, by filling roles of Teaching Assistants, delivering research seminars and mentoring junior students.

\section{Summary and Conclusions}

Our overall goal is to develop and deliver a long-term, sustainable, scientific and cultural outreach program for youth in the Western Newfoundland region (Canada), especially rural youth, girls and Indigenous students, and to create an engaging and inclusive environment for students in our new BSc program in Physics. Combining complementary expertise and resources at MUN's Grenfell Campus, the Qalipu First Nation, Parks Canada and the Newfoundland and Labrador English School District, we feature female and Indigenous role models, engage storytelling, discuss career opportunities, and emphasize a diverse set of skills required in modern science such as creativity, cooperation and communication. Our Qalipu partners are especially instrumental in integrating the Indigenous knowledge in our science curriculum and establishing contacts with schools. Coordinating the engaging science-outreach content with the existing provincial curriculum can be a challenge sometimes, especially since subatomic physics, one of the most fascinating and rapidly developing scientific fields, is not covered in the provincial school curricula. We address that gap by sharing personal research experiences and building on related curriculum topics such as the space unit. In general, we plan to build a scalable and transportable outreach program by starting with local events, visiting schools within driving distance, collecting feedback, evaluating outcomes and building partnerships, and then to expand to more isolated communities in rural Newfoundland and coastal Labrador. It is the most hard-to-reach communities that are in the greatest need of outreach and stimulation, so, in the future, and with resources permitting, we plan to expand to fly-in-only communities and coastal communities reachable only by water. Engaging over a thousand participants per year, our science outreach program would not be possible without Grenfell student volunteers, who are especially instrumental in delivering programs, acting as role models (most volunteers are female), and establishing contacts with high-school teachers. The new BSc program in Physics with a focus on subatomic physics and astrophysics, which graduated its first class in 2015, seems to be quite successful, and we have seen a steady increase in enrollment so far, with some classes quadrupling in size. We aim to provide our undergraduate students with exciting research opportunities, celebrate their successes, and create an engaging, inclusive, and comfortable environment. We are also offering new PhD and MSc programs in theoretical particle physics via MUN St. John's campus, with graduate students contributing to both research and education at Grenfell. While, in the past several decades, Canada has achieved some success in attracting women to sciences in general [15], there is still room for improvement in recruitment and retention of women and other equity-seeking groups in physics, and we believe that subatomic physics specifically, as one of the most fascinating areas of modern research, can help with both attracting and inspiring the next generation of scientists.

\section{Acknowledgments}

This science outreach work is supported by the PromoScience Program of the Natural Sciences and Engineering Research Council of Canada (NSERC). 


\section{References}

[1] CAUT Almanac of Post-Secondary Education in Canada (2010-2011).

[2] Karoline Wiesner, 2002 IUPAP Women in Physics Conference (2002).

[3] Statistics Canada, Census Profile, Census 2016 (2016).

[4] Morgan, C., Isaac, J. D., Sansone, C., "The role of interest in understanding the career choices of female and male college students. Sex Roles", 44, 295-320 (2001).

[5] Patricia H. Miller, Sue V. Rosser, Joann P. Benigno, Mireille L. Zieseniss, "A Desire to Help Others: Goals of High-Achieving Female Science Undergraduates", Vol. 28, No. 1/2, Building Inclusive Science (Spring - Summer), pp. 128-142 (2000).

[6] Eccles, J. S., "Understanding women's educational and occupational choices - Applying the Eccles et. al. model of achievement-related choices", Psychology of Women Quarterly, 18, 585-609 (1994).

[7] Harris, P., Bartlett, C., Marshall, M., Marshall, A., "Mi’kmaq Night Sky Stories; Patterns of Interconnectiveness, Vitality and Nourishment", Communicating Astronomy with the Public Journal (2010).

[8] http://qalipu.ca/

[9] “Grenfell physics students conquer summer job market”, CBC News, March 5 (2014).

[10] “Grenfell student to work on Large Hadron Collider", Western Star, Feb.9 (2014).

[11] “GRENFELL MATTERS: Mallory Snow”, Western Star, Oct. 3 (2018).

[12] M. Bluteau, S. Barkanova, "From CERN to high schools: an argument for greater involvement of postsecondary Physics students in high school outreach”, Physics in Canada, 70.2 (2014).

[13] M. Hewlett, S. Barkanova, "High School Outreach by Postsecondary Students”, Physics in Canada, 73.3 (2017).

[14] M. Ahmady, A. Aleksejevs, S. Barkanova, R. Sandapen, "Particle physics research and education in Atlantic Canada", Physics in Canada, 69.1 (2013).

[15] Li-Hong Xu, S.Ghose, M. Milner-Bolotin, J. McKenna, S. Bhadra, A. Predoi-Cross, A. Dasgupta, M. Campbell, S. Barkanova, M. Steinitz, "Women in Physics in Canada", "Physics in Canada", Special Issue 71 (2015). 